\title{
Advantages of Endosopic Retrograde Cholangiopancreatography with Procedural Sedation and Analgesia Technique
}

\author{
Ljiljana Gvozdenovic*, Mirka Lukic Sarkanovic, Natasa Nestorov, Vladimir Dolinaj, Teodora \\ Bozic \\ Clinical center Vojvodina, Novi Sad, Serbia \\ Medical University Novi Sad, School of medicine, Novi Sad, Serbia
}

*Corresponding Author: Ljiljana Gvozdenovic, Clinical center Vojvodina, Novi Sad, Serbia, Email: ljiljana.gvozdenovic@uns.ac.rs

\begin{abstract}
Objective: Use of day-case anesthesia reduces the amount of medication prescribed, and uses doctor's time more efficiently.

Methods: 330 patients who underwent diagnostic and therapeutic ERCP in PSA, due to certain diseases of pancreatic and biliary system were analyzed using a prospective study. Following data was analyzed using appropriate statistical method: gender, age, indication for ERCP, placement of biliary endoprothesis (stent), as well as appearance of potential postoperative complications.

Results: $86(52 \%)$ were males, and $79(48 \%)$ were females. Based on the age of patients, $29 \%$ of patients were 60 to 70 years of age. Indications for performing ERCP in the remaining 53 patients (32\%) were nonocclusive choledocholithiasis in 52 patients (98\%) and dilatation of hepatocholechus in 1 patient (2\%). In 134 patients (81\%), postoperative period was without complications. They graded the entire work of the anesthesiologist, gastroenterologist and the medical team, as well as their subjective feeling, with the highest grade 10.
\end{abstract}

Conclusions: All patients were exceptionally satisfied, thanks to administration of PSA tehnique.

Keywords: Endoscopic procedure, day-case anesthesia, satisfaction.

\section{INTRODUCTION}

Goal of procedural sedation and analgesia (PSA) is to provide continuous analgosedation for patients undergoing interventional endoscopic procedures, like endosopic retrograde cholangiopancreatography (ERCP) $(1,2)$.

Using a prospective study, 330 patients undergoing diagnostics and therapy via endoscopic retrograde cholangiopancreatography due to certain diseases of pancreatic and biliary tract were evaluated in Emergency Center of Clinical Center of Vojvodina. Control group consisted of 165 patients that were not treated using a PSA technique in day-case clinic because of fear of anesthesia, or because they were urgent cases. Following data was statistically evaluated: gender, age, indications for ERCP, placement of biliary endoprosthesis (stent), and presence of possible postoperative complications. PSA method was provided by intravenous administration of benzodiazepines, and opioid (Fentanyl). For complex and longlasting procedures, total intravenous anesthesia (TIVA), with the administration of nonbarbiturate anesthetic, Propofol, was used.

After the performed procedure, patients filed out an individual list of satisfaction degree, by encircling numbers 1-10 (unsatisfied satisfied), in which they graded the level of satisfaction and subjective comfort with the administered type of anesthesia, as well as the entire work of the anesthesiologist, gastroenterologist and medical staff in the daycase settings (Table 1.).

Out of total number of patients that underwent procedural sedation and analgesia during ERCP intervention in day-case settings $(n=165), 86$ $(52 \%)$ were males and $79(48 \%)$ were females. Based on representation according to age of patients, $29 \%$ of patients were 60 to 70 years of age (47 out of 165 patients). Indications for 
Advantages of Endosopic Retrograde Cholangiopancreatography with Procedural Sedation and Analgesia Technique

ERCP in 55\% were benign causes and in $32 \%$ underwent the procedure due to benign reasons. benign causes. 90 patients $(55 \%)$ were

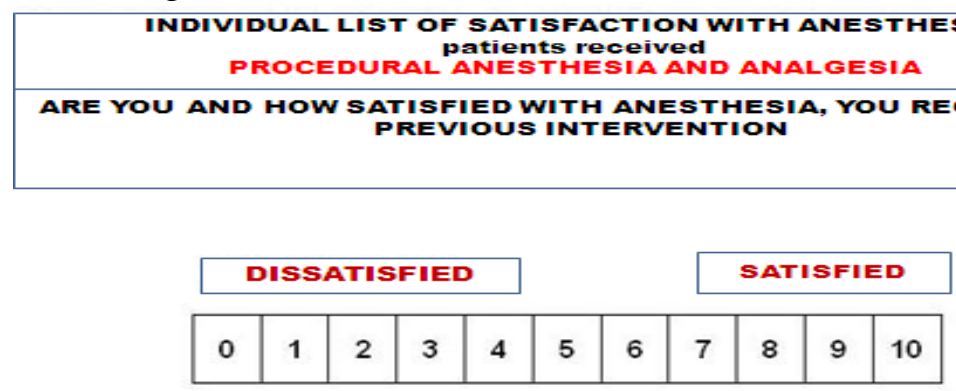

PLEASE CIRCLE THE APPROPRIATE certain scale number that will Point out THE LEVEL OF YOUR PAIN AND ARE YOU FEELING SATISFIED,

Applied Types of anesthesia

Table1. Individual list of patient satisfaction

Biliary endoprosthesis was placed in 22 (13\%) patients. Out of 165 patients that underwent the procedure, complications developed in $4 \%$ patients. Patients graded the entire work of the anesthesiologist, gastroenterologist and medical team, as well their subjective feeling, with the highest grade of 10 . Only $5 \%$ of the patients from the control group reported their satisfaction with the performed ERCP procedure during endoscopic procedures total intravenous anesthesia (TIVA) with the use of nonbarbiturate anesthetic, Propofol, can be administered, as well. Largest clinical study was performed by Trapani G and colleagues (3). Deep sedation was achieved in majority of 9152 patients who underwent long and compex endoscopic procedures. Weaver CS reported a similar series of 2000 patients receiving propofol sedation (4). Hohl CM., provided effectiveness of midazolam versus propofol for 2574 patients undergoing a variety of endoscopic procedures and found a better safety profile of bensodiasepines. Clarke et al. also report a beter safety profile of bensodiasepines in over 28000 patients (5). All patients who were in our study who underwent the endoscopic procedure in day-case settings were exceptionally satisfied, thanks to administration of procedural sedation and analgesia.

\section{REFERENCES}

[1] Verma R, Alladi R, Jackson I.: Day case and short stay surgery: 2, Anaesthesia 2011; 66: 417-434.

[2] von Delius S. Bispectral index monitoring of of midazolam and propofol sedation during endoscopic retrograde cholangiopancreatograpy. Endoscopy. 2012; 44(3):258-64.

[3] Trapani G, Altomare C, Sanna E. Propofol in anesthesia. Mechanism of action, structureactivity relationships, and drug delivery. Curr Med Chem 2007; 7:249-271.

[4] Weaver CS, Hauter WE, Brizendine EJ, Cordell WH. Emergency department procedural sedation with propofol: Is it safe? J Emerg Med. 2007; 33:355-61.

[5] Hohl CM, Sadatsafavi M, Nosyk B, Anis AH. Safety and clinical effectiveness of midazolam versus propofol for procedural sedation in the emergency department: A systematic review. Acad Emerg Med. 2008; 15:1-8.

Citation: Ljiljana Gvozdenovic, Mirka Lukić Sarkanovic, Nataša Nestorov, Vladimir Dolinaj, Teodora Božić. Advantages of Endosopic Retrograde Cholangiopancreatography with Procedural Sedation and Analgesia Technique. ARC Journal of Anesthesiology. 2017; 2(3):3-4. doi: dx.doi.org/10.20431/24559792.0203002.

Copyright: (C) 2017 Authors. This is an open-access article distributed under the terms of the Creative Commons Attribution License, which permits unrestricted use, distribution, and reproduction in any medium, provided the original author and source are credited. 